\title{
Assessing driver's ability to estimate compliance rates to in-car, advisory driver support
}

\author{
Malte Risto • Marieke H. Martens
}

Received: 29 July 2013 / Accepted: 13 December 2013 /Published online: 28 December 2013

(C) The Author(s) 2013. This article is published with open access at SpringerLink.com

\begin{abstract}
Purpose In-car support systems focus increasingly on improving traffic flow and throughput. Advisory systems allow for fast market penetration, advising drivers how to drive in order to improve general flow. By following the advice, drivers cannot create a beneficial effect by themselves but rely on other road users to comply as well. Drivers who sense a low compliance among other road users may be discouraged to use the system themselves. The present experiment investigated whether drivers are able to distinguish between various compliance rates to Connected Cruise Control (CCC), an advisory driver support system that gives headway, speed and lane advice to improve throughput on motorways.

Method Forty-two participants estimated the compliance of other road users to CCC in a driving simulator. Actual system compliance was varied between 10,50 and $90 \%$. Half of the participants received detailed information about the advice and the manifestation of compliant behaviour in traffic.

Results Compliance estimates showed no effect of actual compliance rates. Overall compliance ratings were higher for participants who had not received additional information about the system. Difference scores between compliance estimate and actual compliance indicate that additional information did not improve estimation accuracy, neither did it increase participants' confidence with their estimate.
\end{abstract}

\footnotetext{
M. Risto $(\bowtie) \cdot M$. H. Martens

University of Twente, P.O. Box 2177500 AE Enschede,

The Netherlands

e-mail: m.risto@utwente.nl

M. H. Martens

e-mail: marieke.martens@tno.nl

M. H. Martens

TNO Defence, Security and Safety, P.O. Box 233769 ZG,

Soesterberg, The Netherlands
}

Conclusions When actual compliance is low, drivers still show high compliance estimates which can have beneficial effect on system acceptance. Additional information does not improve compliance estimates.

Keywords Advisory driver support · Traffic flow improvement · Social dilemma · Conditional cooperation . Compliance rate $\cdot$ Estimation

\section{Introduction}

In the past decade innovations in driver support systems have focussed increasingly on the improvement of throughput and traffic flow resulting in systems that aim to improve the distribution and the behaviour of road users on a given road. On the automation spectrum these systems range from more autonomous (i.e. automated) to less autonomous (i.e. advisory) systems [1]. An example of an automated system is a cooperative adaptive cruise control (C-ACC) that uses vehicle-to-vehicle communication to improve string stability $[2,3]$. Advisory systems offer support to drivers who want to improve their driving behaviour while remaining in total control of the vehicle. These systems provide information or advice about the appropriate driving behaviour $[4,5]$. This driver-in-the-loop approach has the advantage that drivers remain in control of the vehicle at all times, thereby eliminating dangerous situations in which the control of the vehicle has to be transitioned between the driver and the vehicle [6]. Also it reduces liability issues that emerge with semi-automated (automated control of part of the driving task) or fully-automated systems (automated control of the complete driving task) in the case of system failure. On the other hand, the effectiveness of these systems is fully dependent on the response of the drivers. If drivers are not willing or able to follow the given advice, the system will not have the intended effect. 
An example of such an advisory system is Connected Cruise Control (CCC; see for example [7, 8]). CCC offers drivers advice on the optimal speed, headway and driving lane in order to optimize the distribution of cars on the motorway and counteract the build-up and propagation of shockwaves. Drivers will receive individual advice messages via an in-car, nomadic device. These individual messages are adjusted to a drivers current lane, headway and speed, the actual speed limit as well as the desired route.

A survey amongst Dutch car drivers investigated the conditions under which drivers would potentially use an advisory driver support system that aims to improve overall traffic flow. It found that one of the strongest incentives for drivers to use such a system (i.e. comply with its advice) would be an observable beneficial effect on traffic flow and throughput [9]. In turn, whether or not such a system has any effect on traffic flow is dependent on the percentage of vehicles on the road that are equipped with the system (i.e. the penetration rate of the system) and the number of drivers that adheres to the given advice (i.e. the compliance rate of drivers).

In case the penetration rate and the compliance rate are sufficiently high, it may still be that the system creates a general benefit for the traffic system but not necessarily for the individual driver who complies with the advice. The outcome of a driver's action is experienced by the collective of road users following the driver. In turn the individual driver is dependent on other drivers in front of him, that are also equipped with the same system, to create a beneficial effect for this driver. As a result, each driver is exposed to the effect of the collective actions of the drivers in front of him. Already in 1971, Schelling articulated how this applies to inconsiderate behaviours that may harm the flow of traffic, by stating: "Unorganized, they [the drivers] are at the mercy of a decentralized accounting system according to which no $[\ldots]$ driver suffers the losses that he imposes on the people behind him" [[10], p.66]. The same mechanisms also apply to efforts to improve traffic flow. It can be questioned, whether the beneficial effect that is created by an individual driver will, in any way, pay itself back to the same driver or whether drivers will ever directly perceive the effect that their compliance has on traffic flow. It may even be that a driver experiences a direct individual disadvantage when following the advice. For example, a driver complies to an advice to merge from a dense middle lane to the right lane. The effect that he creates on traffic flow might be beneficial for other road users, although he now finds himself between trucks on a slow driving right lane. Therefore he might perceive the outcome of his compliance as disadvantageous.

In the same survey, respondents were conscious of this interdependence between road users in creating a collective benefit through the improvement of traffic flow. As a precondition to use the advisory driver support system, they requested that the system should be mandatory, and therefore used by all road users [9]. However, even if a sufficient penetration rate could be guaranteed (for instance through a government mandate to use the system), the potential effect that such a system can have on traffic flow would still be dependent on the collective compliance of a sufficient number of drivers. In other words, to achieve an effect for traffic flow, drivers that use the system are dependent not only on their ability follow an the advice, but also on the ability and willingness of other equipped drivers to comply. Therefore, the interdependency among drivers does not only pose a harm to the improvement of traffic flow, but also to the successful implementation of a system that aims to improve traffic flow. Only when penetration and compliance rate are sufficient, the driver will benefit from collective compliance of other traffic. A challenge for these systems is to generate the necessary compliance rate that will cause a beneficial effect on the whole traffic stream in order to justify the use of the system for the individual drivers.

The situation described here shares similarities with that of a social dilemma [11-13]. Each driver may benefit from improved traffic flow when all road users cooperate by using the system and complying to the advice. However, individually a driver may not gain anything from following the advice and may be better off free-riding (i.e. not following any advice, while benefitting from the effect created by more compliant drivers). Even if drivers are willing to cooperate in the first place, they may fear that the overall compliance rate is not sufficient to actually lead to an improvement of traffic flow. To avoid having the cost of using the system without benefiting from it, due to low overall compliance, drivers may cease to comply themselves.

In order to improve traffic flow, drivers stated to be willing to cooperate, under the condition that others cooperate as well [9]. This refers to a phenomenon also known as conditional cooperation $[14,15]$. A similar idea has been introduced by Pruitt and Kimmel as part of the "goal/expectation theory" [16]. The theory states that cooperative behaviour requires not only an individual's goal to achieve mutual cooperation, "It must be accompanied by an expectation that the other will cooperate [...]." (p. 375). Therefore, in addition to having the goal to improve traffic flow by using CCC, drivers need to be convinced that there is sufficient compliance among other drivers on the road in order to show cooperative (i.e. CCC compliant) behaviour. If drivers are under the assumption that the compliance rate among other drivers is too low, they guard themselves from exploitation by refusing to comply themselves. This makes drivers' ability to detect different levels of compliance, among other road users, an important factor in their own willingness to use the system themselves. If drivers are able to tell a high compliance rate from low a rate, they might be inclined to stop using the system in case of low perceived compliance. Furthermore, if drivers are not able to tell the difference between compliance rates, acceptance of the system would benefit from an overestimation of the compliance rate, especially when the actual compliance rate is low. 
In the present study it was investigated, in a driving simulator, whether drivers are able to deduct the current (or the difference between a high and a low) compliance rate of CCC, from observations of traffic around them. Furthermore, it was tested whether additional information about the system would improve drivers ability to distinguish between different compliance rates or influence the average estimate in any direction.

\section{Method}

\subsection{Participants}

Forty-two participants ( 33 men, 9 women), age 26 to 66 years (M: 53.6, SD: 10.5) completed the experimental procedure. One participant had to abort the experiment due to simulator sickness. All participants were recruited from the pool of participants registered by TNO and had no prior knowledge of the study or the form of driver support used in the experiment. All participants were in possession of a driver's license for at least 4 years (M: 32.7, SD: 11.9) and drove at least 10.000 annual kilometres by car. Participants reported to have normal or corrected to normal vision. Participants received $€ 50$ for their participation.

\subsection{Design}

The experiment, had a $3 \times 3 \times 2$ mixed factorial design with repeated measures. Within-participants variables were location (lane drop vs. on-ramp vs. straight motorway) and actual compliance rate $(10 \%$ vs. $50 \%$ vs. $90 \%)$. Betweenparticipants variable was the level of information (low vs. high) about the driver support system. The dependent variables were the participants estimated compliance rates and their confidence in the estimate.

\subsection{Information about the system}

The driver support system used in this experiment, was the so called Connected Cruise Control. The system gives advice on the optimal speed, headway and driving lane in order to optimize the distribution of cars on the motorway and counteract the build-up and propagation of shockwaves, thereby improving traffic flow and throughput.

Participants were randomly assigned to one of two groups. Both groups received information about the overall goal of the system and the driver-in-the-loop approach that the system takes. Knowledge of this information characterized the low information condition. In addition to that, the high information group received detailed information about the advice given by the system and the goal that is pursued with the advice in the three locations that participants would encounter in the experiment. Furthermore, in a top view, each of the three locations was shown with vehicles on each lane and arrows indicating the driving paths of vehicles that follow a particular advice.

\subsection{CCC advice}

In the experiment each advice was tailored to a particular location and a particular distribution of traffic in that location. The locations that were used in the present experiment (i.e. a lane drop, an on-ramp and a straight motorway) are shown in Fig. 1. A common phenomenon in traffic is an unequal lane distribution of vehicles that can cause disturbance due to over capacity of the left lane [17]. The lane distribution of vehicles near merging zones is also a cause of congestion [18]. The goal of the lane advice is to adjust the distribution of vehicles over the lanes in anticipations of merging vehicles due to the decrease of the number of lanes (i.e. lane drop), the entering of vehicles onto the motorway (i.e. on-ramp), or to alleviate a crowded left lane (i.e. straight motorway). Furthermore, in lane drop locations lane advice is given to drivers on the left lane to stimulate early merging and avoid congestion due to late merging. Speed advice is given in combination with the lane advice by advising drivers to adjust their speed to that of the target lane before merging. Before the lane drop and onramp, headway advice is given to vehicles on the target lane that have not received a lane change advice earlier. Drivers are advised to stay on their lane and extend their headway in order to make room for merging vehicles from the left lane or the on-ramp. Of the potential advice messages that $\mathrm{CCC}$ can provide, the described advice is a subset that is relevant to the locations used in this experiment.

\subsection{Compliance behaviour of other vehicles}

To implement system compliant behaviour of the simulated drivers, compliance zones were defined per lane for every location. In these zones, parameters in the driver model of the simulator were adjusted in a way that the vehicle would exert a behaviour as if following a CCC advice. Figure 1 gives an overview of the specific compliance zones per location.

A parameter in the driver model defined the vehicles urge to carry out an advised behaviour. The urge parameter increased as a virtual vehicle approached the end of a compliance zone. An increased urge resulted in compliant behaviour even in traffic conditions that were not optimal to carry out the advised behaviour. For example, in the case of a lane change advice, as the urge of a specific vehicle to change lanes increased, the vehicle accepted shorter gaps. As a result of this set up, every simulated vehicle that received an advice had carried out the advice at the end of the compliance zone, thereby simulating a compliance rate of $100 \%$. For the experiment it did not matter whether the compliance rate 


\section{LaneDrop OnRamp Straight}

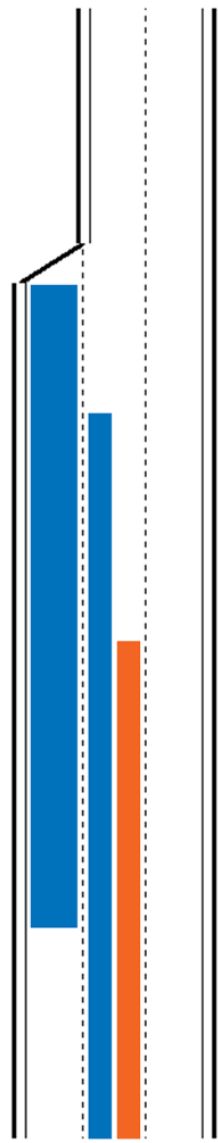

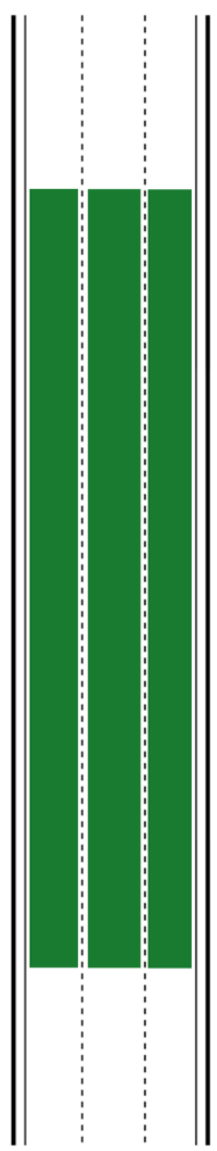

\section{Lane compliance zone \\ Headway compliance zone \\ Optimize compliance zone}

Fig. 1 Compliance zones were used to start and stop the execution of compliance behaviour of the virtual traffic

was kept constant at $100 \%$ while the penetration rate varied between trials or the other way around.

In order to simulate compliance behaviour of equipped vehicles, the standard behaviour model was adjusted as follows:

Lane compliance zone: Upon entry of the lane compliance zone a simulated driver had the urge to change lanes towards the target lane. This urge increased towards the end of the zone.

Headway compliance zone: Upon entry of the headway compliance zone, if the headway of the simulated traffic was smaller than $2 \mathrm{~s}$ it was multiplied by the factor 1.5 and set as the drivers desired headway.

Optimize compliance zone: On the straight motorway, in the beginning, an artificial bottleneck was created by letting the scenario start with an unequal distribution of vehicles over the lanes. The distribution in that scenario was skewed towards the left lane as described in [17]. Upon entry of the optimize compliance zone, equipped vehicles received a lane change advice in order to equalize the distribution of vehicles over the lanes. The number of vehicles that received a lane change advice was computed in such a way that as a result of compliance an equal distribution over the three lanes would be achieved.

\subsection{Driving simulator}

The experiment was conducted in the driving simulator at TNO (Fig. 2). In front of the simulator a radial screen $\left(180^{\circ}\right)$ was present on which the road and the traffic environment were projected with a refresh frequency of $60 \mathrm{~Hz}$. Images for the rear-view and side mirrors were projected on separate screens behind the car. Motor sound as well as the sound of other traffic was presented via loud speakers.

\subsection{Procedure}

Participants were welcomed and asked to read the experiment description and sign the informed consent. Participants then read the group specific information about the CCC system. In the simulator, participants then completed the experimental trials in randomized order. The complete list of trials is shown in Table 1.

After each trial participants were asked to give an estimate of the compliance rate of vehicles in that trial, using a scale from 0 to $100 \%$. Furthermore they rated their confidence with that estimate on a scale from 1 (not at all confident) to 5 (very

Fig. 2 Driving simulator used in the experiment

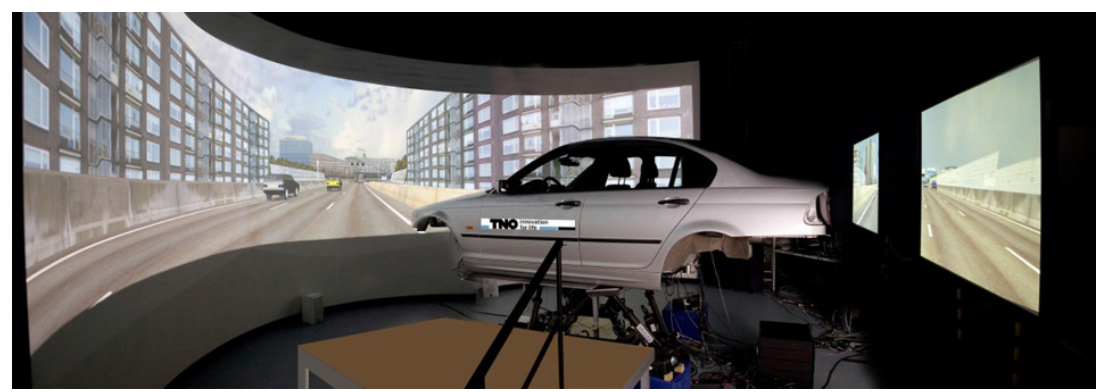


Table 1 Trial list

\begin{tabular}{lll}
\hline Location & $\begin{array}{l}\text { Starting } \\
\text { lane }\end{array}$ & $\begin{array}{l}\text { Compliance } \\
(\%)\end{array}$ \\
\hline Lane drop & Middle & 10 \\
& & 50 \\
& & 90 \\
On ramp & Right & 10 \\
& & 50 \\
Straight motorway & Middle & 10 \\
& & 50 \\
& & 90 \\
\hline
\end{tabular}

confident). After all experimental trials had been completed, participants were debriefed and dismissed.

\subsection{Data analysis}

In addition to the raw estimates of system compliance, absolute estimation errors of the compliance rate (AEEC) were computed per trial by the formula

$A E E C=\sqrt{\left(C_{\text {estimated }}-C_{\text {actual }}\right)^{2}}$

where $C_{\text {estimated }}$ was the compliance rate that was estimated by a participant in that trial and $C_{\text {actual }}$ was the compliance rate that was actually presented to the participant that trial.

Estimated compliance as well as AEEC were analysed in a repeated measures ANOVA with location (lane drop vs. onramp vs. straight motorway) and actual CCC compliance rate (10\% vs. $50 \%$ vs. $90 \%$ ) as within-participant factors and level of information about CCC (low vs. high) as betweenparticipant factor.

Confidence scores were analysed in a repeated measures ANOVA with location (lane drop vs. on-ramp vs. straight motorway) and actual CCC compliance rate (10\% vs. $50 \%$ vs. $90 \%$ ) as within-participant factors and level of information about CCC (low vs. high) as between-participant factor.

As measure of effect size for repeated measures ANOVA, generalized eta squared $\left(\eta_{G}^{2}\right)$ are provided as defined by [19] and recommended by [20]. For differences between means the Pearson Correlation Coefficient (r) is used.

\section{Results}

\subsection{Estimates of Compliance rate}

Figure 3 shows the average estimated compliance in the experiment.

A repeated measures ANOVA was carried out on the estimated compliance scores with location (lane drop vs. on ramp vs. straight motorway) and actual compliance rate (10\% vs. $50 \%$ vs. $90 \%$ ) as within-participant factors and level of information about the system (low vs. high) as betweenparticipant factor.

No main effect of actual compliance on estimated compliance was found $(p<0.05)$. Furthermore, a significant main effect of level of information on estimated compliance was found, $\mathrm{F}(1,40)=6.94, p<0.05, \eta_{G}^{2}=0.084$. However, also the interaction effect between level of information and actual compliance rate on estimated compliance was significant, $\mathrm{F}(2,80)=3.66, p<0.05, \eta_{G}^{2}=0.009$. Figure 4 depicts this interaction.

Post-hoc pairwise comparison with Bonferroni correction revealed that at an actual compliance of $10 \%$, estimated compliance was significantly different between the low information condition (M: 44.05, SE: 4.94) and the high information condition (M: 19.95, SE: 3.77), $\mathrm{t}(124)=4.75, p<0.05, r=$ 0.39 . Also, at an actual compliance of $50 \%$, estimated compliance was significantly different between the low information condition (M: 38.43, SE: 4.71) and the high information condition (M: 22.78, SE: 4.31), $\mathrm{t}(124)=3.00, p<0.05, r=0.26$.
Fig. 3 Average compliance estimates per trial (error bars show the standard error of the mean)

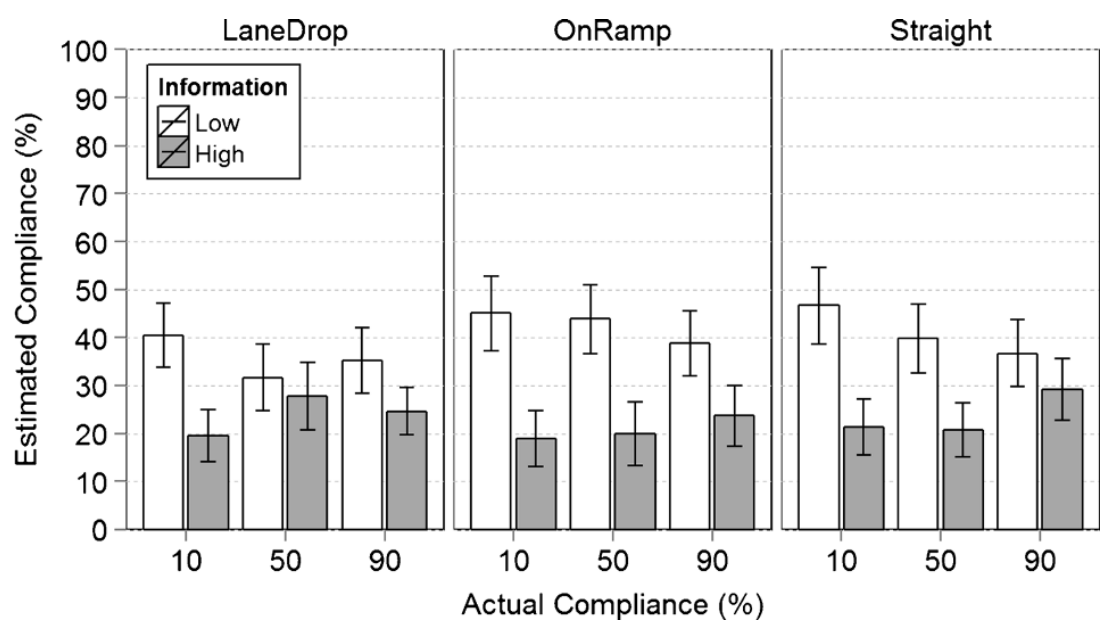




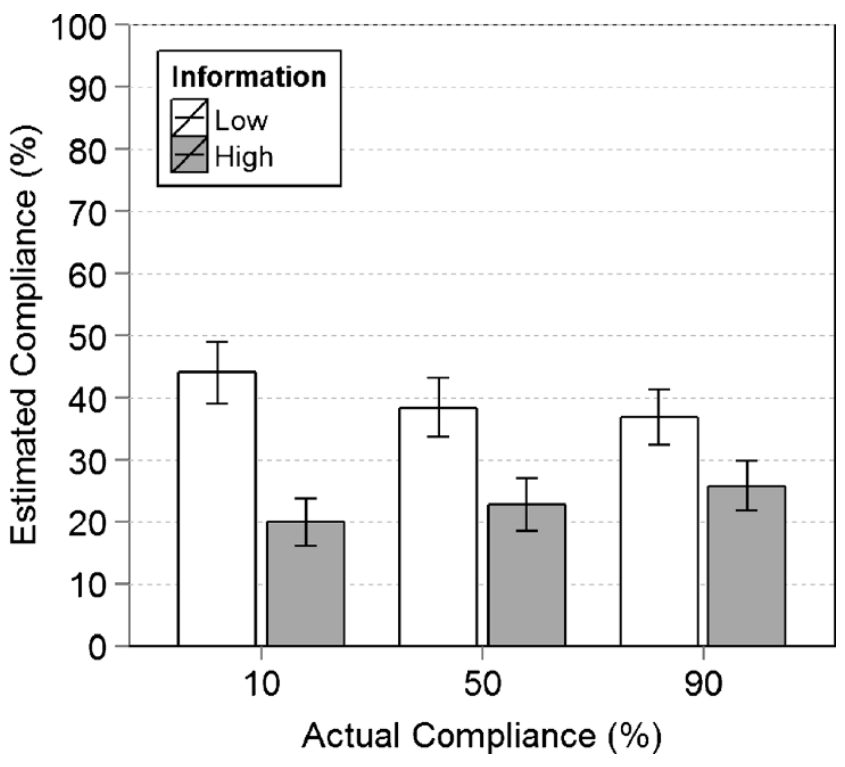

Fig. 4 The interaction effect of level of information and actual compliance rate on the compliance estimate. Error bars show the standard error

The difference in estimated compliance between the low and the high information condition at an actual compliance of $90 \%$ was not significant $(p>0.05)$. Furthermore, mean estimated compliance at each level of actual compliance was not significantly different from estimated compliance at the two other levels of actual compliance, both for the low and the high information condition ( $p>0.05)$.

No other effect was significant at $\alpha=0.05$.

To obtain a measure of the estimation performance, for each compliance estimate the absolute estimation error of the compliance rate (AEEC) was computed according to Eq. (1). Average AEEC provides an indication of the estimation performance in each trial where lower scores denote a better estimation performance (see Fig. 5).

A repeated measures ANOVA was carried out on the AEEC scores with location (lane drop vs. on ramp vs. straight motorway) and actual compliance rate (10\% vs. $50 \%$ vs.
$90 \%$ ) as within-participant factors and level of information about the system (low vs. high) as between-participant factor.

Mauchly's test indicated that the assumption of sphericity had been violated for the main effect of actual compliance rate, $W=0.34, p<0.05, \varepsilon=0.60$, as well as the interaction effect of actual compliance rate and level of information $W=0.34$, $p<0.05, \varepsilon=0.60$. For these effects the degrees of freedom were adjusted using Greenhouse-Geisser estimates of sphericity.

A significant main effect of actual compliance rate on AEEC was found, $\mathrm{F}(1.2,48)=30.83, p<0.05, \eta_{G}^{2}=0.271$. Also the interaction effect between actual compliance rate and level of information was significant, $\mathrm{F}(1.2,48)=8.33$, $p<0.05, \eta_{G}^{2}=0.091$. Figure 6 depicts this interaction.

Post-hoc pairwise comparison with Bonferroni correction revealed that at an actual compliance of $10 \%$, AEEC was significantly different between the low information condition (M: 37.86, SE: 4.21) and the high information condition (M: 17.03, SE: 3.09), $\mathrm{t}(113.80)=4.88, p<0.05, r=0.42$. The difference in estimated compliance between the low and the high information condition at an actual compliance of $50 \%$ and at $90 \%$ was not significant $(p>0.05)$. Furthermore, for the low information condition, mean AEEC at $50 \%$ actual compliance (M: 28.56, SE: 2.39) was significantly different from AEEC at $90 \%$ actual compliance (M: 53.10, SE: 4.49), $\mathrm{t}(62)=-8.00$, $p<0.05, r=0.71$. For the high information condition, mean AEEC at $10 \%$ actual compliance (M: 17.03 , SE: 3.09) was significantly different from AEEC at $50 \%$ actual compliance (M: 35.95, SE: 2.29), $\mathrm{t}(62)=-5.71, p<0.05, r=0.59$. Also, mean AEEC at $50 \%$ actual compliance was significantly different from AEEC at $90 \%$ actual compliance (M: 64.18, SE: 3.92), $\mathrm{t}(62)=-9.78, p<0.05, r=0.78$.

No other effect was significant at $\alpha=0.05$.

\subsection{Confidence with the compliance estimate}

Participants' confidence with their estimate per scenario is shown in Fig. 7. Recall that participants rated the confidence
Fig. 5 The absolute estimation error of the compliance rate (AEEC) per trial. Error bars show the standard error

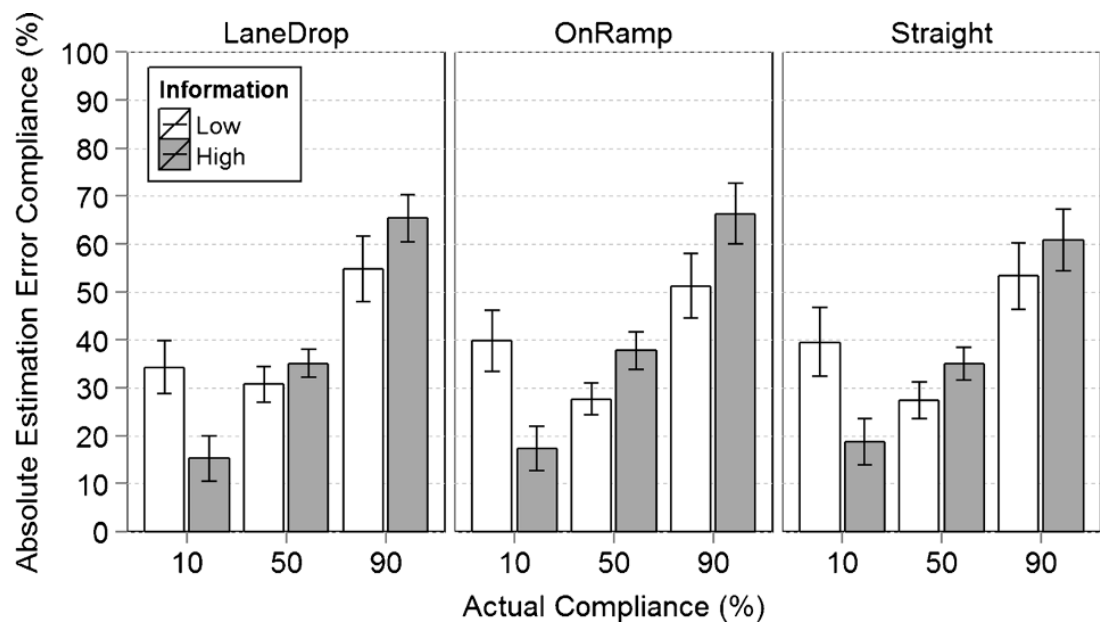




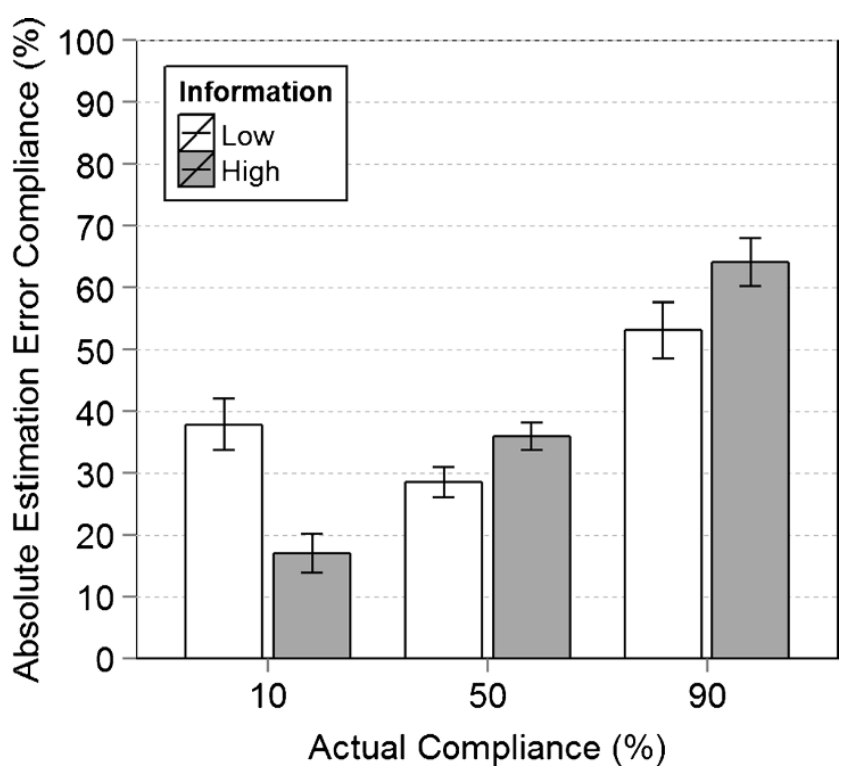

Fig. 6 The interaction effect between compliance rate and level of information on the AEEC. Error bars show the standard error

with their compliance estimate on a scale from 1 (not at all confident) to 5 (very confident).

A repeated measures ANOVA was carried out on the confidence scores with location (lane drop vs. on ramp vs. straight motorway) and actual compliance rate (10\% vs. $50 \%$ vs. $90 \%$ ) as within-participant factors and level of information about the system (low vs. high) as between-participant factor.

No effect was significant at $\alpha=0.05$.

\section{Discussion}

The present study examined driver's ability to distinguish between different compliance rates to CCC, an advisory driver support system aimed at improving traffic flow. Furthermore it was investigated whether additional information could improve or in other ways alter drivers estimates of how many other car drivers complied to advice from the CCC system. No main effect of actual compliance rate on participants estimate of compliance was found. However a significant main effect of the level of information as well as an interaction effect of level of information and actual compliance was found. Participants in the high information condition estimated compliance to be lower than participants in the low information condition. This difference appears to decrease with rising levels of actual compliance rate. More informed participants had received explicit information about the advice that the system would give in a given location and had therefore a better understanding of the behaviour that the traffic around them would exert at higher levels of compliance to the system as well as a top view of coordinated behaviour patterns that groups of equipped vehicles would show at higher compliance rates. In contrast participants in the low information condition had only received information about the system's general aim and that a driver-in-the-loop approach is used. Therefore, drivers in the low information condition might have looked at traffic behaviour more generally, while drivers in the high information condition might have looked for particular indicators of compliance that they had learned of during the introduction to the experiment. Guided by the advice algorithm, equipped vehicles showed a different driving behaviour, compared to the standard driver model of the TNO simulator. However, it appears that drivers failed to distinguish CCC compliant behaviour from regular driving behaviour. Traffic in general was flowing most of the time regardless of the simulated compliance rate. Although occasionally traffic flow was disturbed near the lane drops, this did not lead to traffic jams. Traffic flow would recover from these disturbances so that drivers would not end up in congestion. Uninformed drivers may have credited the general lack of congestion to an elevated compliance rate and therefore gained a more optimistic view of compliance than informed drivers. What can be seen as advantageous for the acceptance of the system is that drivers overestimate others compliance to $\mathrm{CCC}$ advice when the actual compliance rate is low (i.e. $10 \%$ ).
Fig. 7 Confidence level with estimated compliance rates. Error bars show the standard error

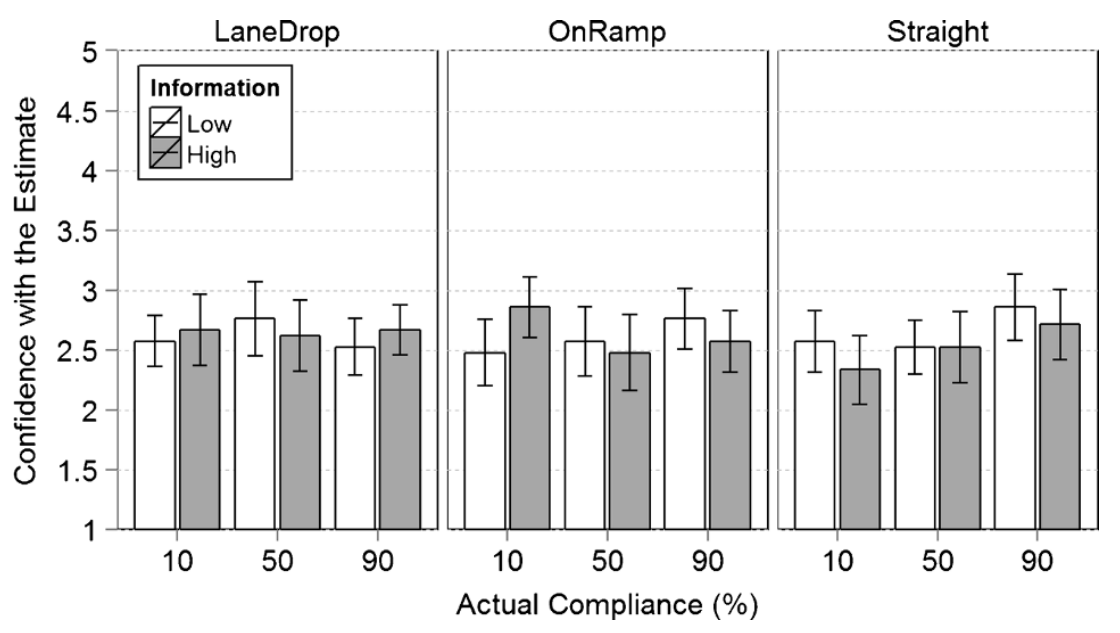


No main effect of the level of information on estimation performance (indicated by the AEEC) was found. There was a main effect of actual compliance level on AEEC. For higher levels of actual compliance the difference scores with estimated compliance increased as well. This is led to the higher AEEC. It suggests, that estimation performance decreased (higher AEECs) with rising levels of actual compliance. Also an interaction effect of compliance level and level of information on AEEC was found. This interaction effect stems from the elevated AEEC of the low information group at $10 \%$ actual compliance compared to the low AEEC of the high information group (see Fig. 6).

Participants' ratings of confidence with their compliance estimates showed no effect of actual compliance rate, information level or location, while average confidence was between 2 and 3. These results suggest that the additional information did not help to improve participants' average levels of confidence in their estimates. Still, the average confidence rating appear high given the low estimation performances. Participants on average were neither extremely confident nor extremely unconfident about their estimates. This level of confidence paired with low actual performance might hint at the difficulty of the task of estimating compliance rate, where even estimates given with high levels of confidence turned out to be wrong.

The present results support the notion that, when implementing systems whose beneficial effect depends on perceived compliance rate, drivers should not be provided with additional information about the functioning of the system. Additional information lowers compliance estimates, while having no effect on drivers estimation performance or confidence with the estimate. Yet, informed drivers may be less likely to comply to an advisory system as they are more likely to perceive the compliance of other road users as low. Furthermore, it shows that uninformed drivers estimate compliance as high even in situations where actual compliance is low (i.e. $10 \%$ ). This can benefit the acceptance of systems where the interdependence between drivers is crucial to their successful implementation.

The participants in the present study were on average above 50 years old and had a good amount of driving experience. Therefore, a generalisation of the data to a population of younger, less experienced drivers should be made with caution. These drivers, may react differently to the presented information and may give different confidence ratings with their estimates. Further research may show whether these results are also obtained with inexperienced drivers.

Acknowledgments This research was conducted in the Connected Cruise Control project, sponsored by Agentschap NL as a High Tech Automotive Systems (HTAS) project.

Open AccessThis article is distributed under the terms of the Creative Commons Attribution License which permits any use, distribution, and reproduction in any medium, provided the original author(s) and the source are credited.

\section{References}

1. Flemisch F, Kelsch J, Löper C et al (2008) Automation spectrum, inner/outer compatibility and other potentially useful human factors concepts for assistance and automation. In: de Waard D, Flemisch FO, Lorenz B et al (eds) Human factors for assistance and automation. Shaker Publishing, Maastricht, pp 1-16

2. Van den Broek THA, Ploeg J, Netten BD (2011) Advisory and autonomous cooperative driving systems. IEEE International Conference on Consumer Electronics (ICCE) 279-280. doi: 978-14244-8712-7/11

3. Ploeg J, Serrarens AFA, Heijenk GJ (2011) Connect \& drive: Design and evaluation of cooperative adaptive cruise control for congestion reduction. J Mod Transp 19:207-213. doi:10.3969/j.issn.2095-087X. 2011.03.009

4. Van den Broek THA, Netten BD, Hoedemaeker M, Ploeg J (2010) The experimental setup of a large field operational test for cooperative driving vehicles at the A270. 13th International IEEE Conference on Intelligent Transportation Systems 198-203. doi: 10. 1109/ITSC.2010.5625050

5. Happee R, Saffarian M, Terken J, et al. (2011) Human factors in the connect \& drive project. Proceedings of the 8th International Automotive Conference

6. Van den Beukel AP, van der Voort MC (2011) Human-centered challenges and contributions for the implementation of automated driving. In: Meyer G, Valldorf J (eds) Advanced microsystems for automotive applications 2011. Springer Berlin Heidelberg, Berlin, pp 225-235

7. Schakel W, van Arem B, van Nes R (2010) Connected cruise control, an advisory system for efficient traffic flow. 11th Trail Congress Connecting People - Integrating Expertise

8. Schakel W, van Arem B (2013) Improving traffic flow efficiency by In-car advice on lane, speed and headway. The 92nd Annual Meeting of the Transportation Research Board

9. Risto M, Martens MH (2011) User acceptance of the connected cruise control and driver irritation in dense motorway traffic. CCC Project Deliverable 6.1a. 1-18.

10. Schelling TC (1971) On the ecology of micromotives. The public interest 25:61-98

11. Dawes RM, Messick DM (2000) Social dilemmas. Int J Psy 35:111116. doi: $10.1080 / 002075900399402$

12. Kollock P (1998) Social dilemmas: The anatomy of cooperation. Annu Rev Sociol 24:183-214

13. Dawes RM (1980) Social dilemmas. Annu Rev Psy 31:169-193

14. Keser C, van Winden F (2000) Conditional cooperation and voluntary contributions to public goods. Scand J Econ 102:23-39. doi:10. 1111/1467-9442.00182

15. Fischbacher U, Gachter S, Fehr E (2001) Are people conditionally cooperative ? Evidence from a public goods experiment. Econ Lett 71:397-404

16. Pruitt DG, Kimmel MJ (1977) Twenty years of experimental gaming: Critique, synthesis, and suggestions for the future. Annu Rev Psy 28: 363-392. doi:10.1146/annurev.ps.28.020177.002051

17. Schakel W, Knoop VL, van Arem B (2012) LMRS: An integrated lane change model with relaxation and synchronization. Trans Res Rec 47-57

18. Knoop VL, Duret A, Buisson C, van Arem B (2010) Lane distribution of traffic near merging zones influence of variable speed limits. 13th International IEEE Conference on Intelligent Transportation Systems. Ieee, Madeira, Portugal, pp 485-490

19. Olejnik S, Algina J (2003) Generalized eta and omega squared statistics: Measures of effect size for some common research designs. Psy method 8:434-447. doi:10.1037/1082-989X.8.4.434

20. Bakeman R (2005) Recommended effect size statistics for repeated measures designs. Behav Res Method 37:379-384 